\title{
Characterization of high-finesse mirrors: Loss, phase shifts, and mode structure in an optical cavity
}

\author{
Christina J. Hood and H. J. Kimble \\ Norman Bridge Laboratory of Physics 12-33, California Institute of Technology, Pasadena, California 91125 \\ Jun Ye \\ JILA, National Institute of Standards and Technology, University of Colorado, Boulder, Colorado 80309
}

(Received 27 January 2001; published 13 August 2001)

\begin{abstract}
An extensive characterization of high-finesse optical cavities used in cavity QED experiments is described. Different techniques in the measurement of the loss and phase shifts associated with the mirror coatings are discussed and their agreement shown. Issues of cavity-field mode structure supported by the dielectric coatings are related to our effort to achieve the strongest possible coupling between an atom and the cavity.
\end{abstract}

DOI: 10.1103/PhysRevA.64.033804

\section{INTRODUCTION}

For many contemporary physics experiments, measurement enhancement via an optical cavity is a useful tool. Indeed, an optical cavity allows one to extend the interaction length between matter and field, to build up the optical power, to maintain a well-defined mode structure, and to study the extreme nonlinear optics and quantum mechanics associated with the large field of a single photon for small cavity volumes [1]. In most situations, a better understanding of cavity and mirror properties is important for achieving improved sensitivity and for elimination of systematic errors. For example, in cavity QED, one needs to know the mode structure of the intracavity field in order to develop the optimum strategy of atom-cavity coupling; for frequency metrology, accurate determination of phase shifts of the resonant fields can provide precision frequency markers; and in quantitative spectroscopy, knowledge of the mirror loss sets the accuracy scale of absorption measurement. On the technology development side, the knowledge gained from careful mirror characterization could provide guidelines for the optic coating community to develop in situ measurement and control capabilities of the coating process.

The work presented in this paper is motivated by the everincreasing demand for a high coherent coupling rate between an atom and the field, as well as of a decreasing cavity loss rate. The aim is to have coherent (reversible) evolution dominating over dissipative processes, and thereby to explore manifestly quantum dynamics in real time, which in turn should lead eventually to the investigation of the strong conditioning of system evolution on measurement results and the realization of quantum feedback control. An important feature associated with strong coupling is that system dynamics are readily influenced by single quanta. Thus singleatom and single-photon cavity QED provides an ideal stage where the dynamical processes of individual quantum systems can be isolated and manipulated. A collection of such coherent systems could help to realize a distributed quantum network for computation and communication [2]. At each node, the quantum information is stored by one or a collection of entangled atoms. Photons serve as the communication link, which in turn entangle the whole network. Within this context, technical advances in optical cavity quantum elec-
PACS number(s): 42.60.Da, 42.55.Sa, 42.79.Gn, 42.79.Wc

trodynamics have become increasingly important. Some significant developments along these lines have been achieved by the group at Caltech [3-8] as well as by other groups $[9,10]$. In Ref. [8], the one-photon Rabi frequency is $\Omega_{1} / 2 \pi=220 \mathrm{MHz}$, in comparison with the atomic decay rate $\gamma_{\perp}=2.6 \mathrm{MHz}$ and the cavity decay rate $\kappa / 2 \pi$ $=14.2 \mathrm{MHz}$.

The strong-coupling condition $\Omega_{1} \gg\left(\gamma_{\perp}, \kappa\right)$ is achieved by using a small cavity length, of the order of $10 \mu \mathrm{m}$. Precise measurement of the length of a short optical cavity facilitates the determination of mirror-coating characteristics. A $10 \mu \mathrm{m}$ cavity length translates to a free spectral range (FSR) of $15 \mathrm{THz}$, or a wavelength difference of a few tens of nanometers (for example, it is $36 \mathrm{~nm}$ for a center wavelength of $852 \mathrm{~nm}$ ) for neighboring cavity modes. Therefore, a straightforward six-digit measurement of the wavelengths (Burleigh wavemeter) of the cavity modes acquires a precision of the order of $5 \times 10^{-5}$ for accurate determination of the equivalent optical length of the cavity, from which details of the index of refraction and layer thickness of materials in the mirror stack can be inferred.

The low loss rate of the cavity field is made possible by high-quality mirror coatings that lead to scatter and absorption losses in the $10^{-6}$ range $[11,12]$. The cavity finesse and overall cavity transmission can be measured directly to determine the mirror losses, $l$, and transmission, $T$. This information can be combined with the FSR measurement in two useful ways: First, the FSR measurement is sensitive to the difference in refractive index $n_{H}-n_{L}$ of the materials making up the multilayer mirror stack, whereas the transmission $T$ depends on the ratio $n_{H} / n_{L}$, as will be shown later. As a result, a precise measurement of both the FSR and $T$ can be used to determine the values of $n_{H}$ and $n_{L}$ independently. Moreover, by mapping out the wavelength dependence of the FSR, the thickness of layers in the mirror stack can be determined. Second, if one of the refractive indices (here $n_{L}$ ) is well known, then the FSR measurement determines $n_{H}$, and an independent value for the mirror transmission $T$ can then be calculated from $n_{H}$ and $n_{L}$, and compared to the experimentally measured result. Indeed, the work presented in this paper shows that we are able to make complementary and mutually confirming measurements of the cavity properties by the two approaches, i.e., measurements of the direct cav- 
ity loss and the dispersion of the cavity modes.

Coming back to the cavity QED experiments, we note that knowledge of the cavity properties is of importance in two particular ways. (i) Mirror absorption/scatter losses are a critical limiting factor in the loss rate from our cavity QED system: for our current cavities the loss rate from photon scattering due to mirror imperfections is similar in size to the atomic spontaneous-emission rate. To build robust quantum computing/communication devices from cavity QED components, it is necessary to improve the ratio of mirror transmission to mirror losses. (ii) The standing-wave light field inside the cavity penetrates into the mirror coatings, giving a larger mode volume $V_{\text {mode }}$ than would be expected naively from the physical distance between the mirror surfaces. Since $\Omega_{1}$ $\propto 1 / \sqrt{V_{\text {mode }}}$, as our micro-cavities are pushed to shorter lengths, this leakage field will have a non-negligible effect on the achievable coupling strength $g_{0}=\Omega_{1} / 2$.

\section{DIRECT TRANSMISSION AND LOSS MEASUREMENTS}

All of the mirrors described in this paper were fabricated by Research Electro-Optics in Boulder, Colorado [12]. More specifically, the measurements were made for the particular coating run REO no. T95 and involved mirrors with radius of curvature $R=10$ and $20 \mathrm{~cm}$. The coating run had a design transmission of $T^{\text {th }}=7 \mathrm{ppm}$ at a center wavelength of 852 $\mathrm{nm}$, from which a cavity finesse of $\mathcal{F}=370000$ was expected. It was somewhat surprising, therefore, to measure a finesse of $\mathcal{F}=480000$ at the targeted wavelength, and this prompted us to make more detailed measurements of the mirror properties and design a model to match these measurements.

First, losses were measured directly with an approximately $40 \mu \mathrm{m}$ length cavity of $20 \mathrm{~cm}$ radius of curvature mirrors in the usual way by recording resonant cavity transmission, reflection, and finesse. If we denote the transmission of mirrors 1 and 2 by $T_{1}$ and $T_{2}$, respectively, and the (absorption + scatter) loss per mirror as $l_{i}=(A+S)_{i}$, then the total cavity losses $\mathcal{L}=T_{1}+T_{2}+l_{1}+l_{2}$ can be determined from the cavity finesse $\mathcal{F}$, given by (FSR) $/ 2 \kappa$, with FSR as the cavity free spectral range and $\kappa$ as the half-width at half maximum for the $\mathrm{TEM}_{00}$ mode of the cavity; equivalently, $\mathcal{F}=2 \pi / \mathcal{L}$. The cavity linewidth $\beta=2 \kappa$ can be determined from a ringdown measurement or using a modulation sideband as a frequency marker with the cavity length scanned, which is the technique employed here. The cavity transmission $I_{\text {trans }}=4 T_{1} T_{2} /\left(T_{1}+T_{2}+l_{1}+l_{2}\right)^{2}$ can then be used to determine $l_{1}+l_{2}$, if $T_{1}$ and $T_{2}$ are known independently. In practice, this is a difficult measurement to make, because the overall transmission $I_{\text {trans }}$ depends on the mode matching into the cavity being perfect. A variation of this protocol that does not require perfect mode matching can be derived by comparing the cavity reflection and transmission values with the cavity locked on resonance and off resonance.

The rudiments of this protocol are as follows. First of all, the total loss $\left(\mathcal{L}=T_{1}+T_{2}+l_{1}+l_{2}\right)$ is always measured first with the determination of the cavity FSR and linewidth. Now let us denote the input power as $P_{\text {in }}$, the reflected power as $P_{r}$, and the transmitted power as $P_{t}$. There is also a mode- matching factor $\epsilon$, meaning that of the input power of $P_{\text {in }}$, only $\epsilon P_{\text {in }}$ is useful for coupling to the cavity $\mathrm{TEM}_{00}$ mode; $(1-\epsilon) P_{\text {in }}$ is wasted. We have the following equations (the assumption of two equal mirrors is reasonable since the two mirrors are produced in the same coating run):

$$
\begin{gathered}
\mathcal{F}=\frac{2 \pi}{T_{1}+T_{2}+l_{1}+l_{2}}=\frac{\pi}{l+T} \\
\frac{P_{t}}{\epsilon P_{\text {in }}}=4 T_{1} T_{2}\left(\frac{\mathcal{F}}{2 \pi}\right)^{2}=T^{2}\left(\frac{\mathcal{F}}{\pi}\right)^{2} \\
\frac{P_{r}-(1-\epsilon) P_{\text {in }}}{\epsilon P_{\text {in }}}=\left(l_{1}+l_{2}+T_{1}-T_{2}\right)^{2}\left(\frac{\mathcal{F}}{2 \pi}\right)^{2}=l^{2}\left(\frac{\mathcal{F}}{\pi}\right)^{2} .
\end{gathered}
$$

Remember that $(1-\epsilon) P_{\text {in }}$ is the "useless" power that is reflected directly off of the input mirror, and must be subtracted from $P_{r}$ to leave the reflected power we wish to measure, that is, the sum of the field leaked from the cavity storage and the field (mode-matched) directly reflected off the input mirror. This cavity contrast is a direct result of the mirror properties. Division of Eq. (2.2) by Eq. (2.3) gives

$$
\frac{P_{t}}{P_{r}-P_{\text {in }}}=\frac{T^{2}\left(\frac{\mathcal{F}}{\pi}\right)^{2}}{l^{2}\left(\frac{\mathcal{F}}{\pi}\right)^{2}-1} .
$$

Equation (2.4), combined with Eq. (2.1), will determine completely $T$ and $l$.

In the actual experiment, this direct measurement approach found that (from finesse we have $l+T$ $=7.2 \mathrm{ppm}) P_{\text {in }}=54 \mu \mathrm{W}, \quad P_{r}=42.6 \mu \mathrm{W}, \quad$ and $P_{t}$ $=4.82 \mu \mathrm{W}$ and therefore $l=2.9 \mathrm{ppm}$ and $T=4.3 \mathrm{ppm}$, with measurement uncertainties below $5 \%$.

Another way to measure the $(T, l)$ is by sweeping out all the high-order spatial modes and carefully noting the transmission and reflection powers at each spatial mode. One measures the total input power and also sums together the powers of every matched mode for transmission and reflection. These three powers can be used in Eqs. (2.2) and (2.3) to calculate the partition between $T$ and $l$. That measurement produced $l=3 \mathrm{ppm}$ and $T=4.2 \mathrm{ppm}$. The value of $T$ should be a bit lower in this case because it is not possible to include all higher-order modes in the measurement; some of them are simply impossible to resolve due to their weakness.

Other cavities measured with mirrors from the same coating run had higher finesse (within 15\%), very likely due to a lower density of surface defects. To construct a cavity of minimal mode volume for the intended maximal coherent coupling rate, we need to have the distance between two mirrors (radius of curvature $R=10 \mathrm{~cm}$ ) on the order of $10 \mu \mathrm{m}$ or below. To avoid contact between the outer edges of the two mirrors, the mirrors were fabricated with coneshaped fronts, reducing the substrate radius from $3 \mathrm{~mm}$ to 1 $\mathrm{mm}$. We notice this extra machine process might have introduced some additional surface defects on some mirrors. 
However, the highest finesse achieved with cone-shaped mirrors was comparable to unmodified pieces, at $\mathcal{F}=480000$ \pm 10000 , corresponding to losses $l=2.2 \mathrm{ppm}$ if mirror transmission $T=4.3 \mathrm{ppm}$ as determined from the above measurements.

\section{TECHNICAL DETAILS OF THE MODEL}

In this section, we derive a model for the coating properties. A transfer-matrix formalism was used to calculate the input-output propagation of a plane-wave field through the 37-layer stack of alternating high-index $\left(\mathrm{Ta}_{2} \mathrm{O}_{5}, n_{H}\right.$ $=2.0411)$ and low-index $\left(\mathrm{SiO}_{2}, n_{L}=1.455\right)$ dielectric layers (the variations of these dielectric constants are within $0.5 \%$ across the entire wavelength region of $650-1060 \mathrm{~nm}$ and about $0.1 \%$ for the interested region of $800-900 \mathrm{~nm}$ and are therefore assumed to be constant for the present precision level). The substrate refractive index (supplied by REO) used was $n_{\text {sub }}=1.5098$. That is, the transfer of the field through each $\lambda / 4$ layer is represented by a matrix, and the response of the entire mirror (or cavity) is determined by the product of these individual matrices. Please note that the typical cavity length used in our experiment is on the order of 1/100 of the confocal parameter (twice the Rayleigh range) of the cavity field, hence justifying the plane-wave model as an excellent approximation.

Following the treatment of Hecht [13] for normal incidence, we take the matrix representing layer $j$ to be given by

$$
M_{j}=\left[\begin{array}{cc}
\cos \left(k h_{j}\right) & {\left[i \sin \left(k h_{j}\right)\right] / Y_{j}} \\
i Y_{j} \sin \left(k h_{j}\right) & \cos \left(k h_{j}\right)
\end{array}\right] .
$$

Here $M_{j}$ relates the electric and magnetic fields $(E, H)$ of the input and output via

$$
\left[\begin{array}{c}
E_{\text {out }} \\
H_{\text {out }}
\end{array}\right]=[M]\left[\begin{array}{c}
E_{\text {in }} \\
H_{\text {in }}
\end{array}\right] .
$$

$k=2 \pi / \lambda$ is the free-space wave vector of the incident light, $h_{j}=n_{j} x$ (layer thickness) with $n_{j}$ the refractive index, and $Y_{j}=\sqrt{\left(\epsilon_{0} / \mu_{0}\right)} n_{j}$ with $\left(\epsilon_{0}, \mu_{0}\right)$ the electric and magnetic constants in SI units. For an exact $\lambda / 4$ layer (and for light at the design wavelength of the coating), this simplifies to

$$
M_{j}=\left[\begin{array}{cc}
0 & i / Y_{j} \\
i Y_{j} & 0
\end{array}\right] \text {. }
$$

A multilayer stack is represented by multiplying the matrices of the individual layers: For light incident on layer 1, the matrix for the entire structure of $q$ layers is defined as the product $M=M_{1} M_{2} \cdots M_{q}$. For our mirror stack, this gives $M=\left(M_{\mathrm{Ta}_{2} \mathrm{O}_{5}} M_{\mathrm{SiO}_{2}}\right)^{18} M_{\mathrm{Ta}_{2} \mathrm{O}_{5}}$. Note that at the coating center (where there is an exact $\lambda / 4$ layer),

$$
M_{\mathrm{Ta}_{2} \mathrm{O}_{5}} M_{\mathrm{SiO}_{2}}=\left[\begin{array}{cc}
-\frac{n_{L}}{n_{H}} & 0 \\
0 & -\frac{n_{H}}{n_{L}}
\end{array}\right],
$$

so the system matrix has the simple form

$$
M=\left[\begin{array}{cc}
0 & \frac{i}{Y_{H}}\left(\frac{n_{L}}{n_{H}}\right)^{18} \\
i Y_{H}\left(\frac{n_{H}}{n_{L}}\right)^{18} & 0
\end{array}\right] .
$$

For a field incident from material with index $n_{0}$ and exiting into material with index $n_{s}$, the resulting transmission coefficient is given by

$$
t=2 Y_{0} /\left(Y_{0} M_{11}+Y_{0} Y_{S} M_{12}+M_{21}+Y_{S} M_{22}\right),
$$

with transmission $T=n_{s} / n_{0}|t|^{2}$ (the factor $\sqrt{n_{s} / n_{0}}$ accounts for the change in the amplitude of the electric field in the dielectric, thereby conserving the net energy flux). At the center wavelength of the coating, then,

$$
T=\frac{n_{s}}{n_{0}}\left|-2 i /\left[\left(n_{S} / n_{H}\right)\left(n_{L} / n_{H}\right)^{18}+\left(n_{H} / n_{0}\right)\left(n_{H} / n_{L}\right)^{18}\right]\right|^{2} .
$$

We can make a further simplification: as $\left(n_{L} / n_{H}\right)^{18}$ $=0.0018$ and $\left(n_{H} / n_{L}\right)^{18}=557$, the first term in the denominator of the above equation is only a $10^{-6}$ correction, so the final result for $T$ at the coating center becomes

$$
T=4 n_{S} n_{0}\left(n_{L}\right)^{36} /\left(n_{H}\right)^{38},
$$

and the transmission is determined by the ratio of the refractive indices.

This calculation reproduced the target reflectivity of $T^{\text {th }}$ $=7.3 \mathrm{ppm}$ for the coating run no. T95 and $T^{\text {th }}=14.6 \mathrm{ppm}$ for another REO coating run no. D1306, where the number of layers was reduced to 35 . The model and measured (REO spectrophotometer data) "coating curves" are shown in Fig. 1 for the no. D1306 coating run.

For a fixed cavity length, the resonance wavelengths of the cavity can be calculated simply with the same transfermatrix formalism, using a matrix for the entire system, $M_{\text {total }}=M M_{\text {gap }} M$ (a product of two mirrors plus a fixedlength vacuum gap in between). The calculation steps through a series of wavelengths calculating the cavity transmission $T$ at each, and by finding places of maximum transmission it finds the vacuum wavelengths of the cavity resonances.

Conversely, for a given set of measured cavity-resonance wavelengths, it is possible to determine the effective cavity length precisely. With a commercial wavelength meter that gives six-digit wavelength measurement, we typically measure the cavity resonance within an uncertainty of $0.01 \mathrm{~nm}$. Error propagation analysis gives an uncertainty for the determination of the effective cavity length (tens of microns) on the order of $0.05-0.1 \mathrm{~nm}$. The parameters of the model (index contrast, layer thickness) are set by comparison to such measurements. Hence, armed with the detailed knowledge of the mirrors provided from the model, the physical cavity length can be determined precisely from a single measurement of resonance, for example when the cavity is 

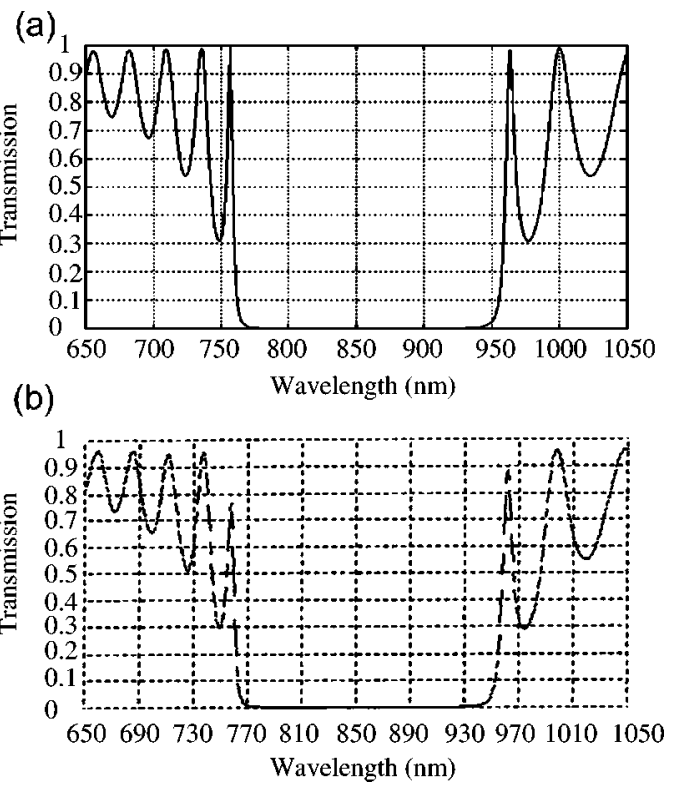

FIG. 1. (a) Calculated and (b) measured transmission of coating as a function of wavelength, for a 35 layer $\lambda / 4$ stack with $n_{H}$ $=2.0411, n_{L}=1.455$, and center wavelength $850 \mathrm{~nm}$. The measured data were obtained in a spectrophotometer operated at REO for the coating run no. D1306.

locked to a laser of known frequency (in our case, a cesium transition at $852.359 \mathrm{~nm}$ ). Close to the center of the design wavelength of the coating, the effective cavity length (on resonance) is roughly $L_{\text {eff }}=L+1.633 \lambda / 2$ with $L$ (the physical distance between the mirror surfaces) an integer number of $\lambda / 2$. The physical cavity length can therefore be determined, with an uncertainty of $\sim 0.5 \mathrm{~nm}$, limited by the overall parameter fitting in the model. Further details of the wavelength dependence are provided by reference to the model.

\section{FREE SPECTRAL RANGE (FSR) MEASUREMENTS}

To determine the parameters of the model (index contrast, layer thickness), a series of precise measurements of the cavity FSR (frequency between successive cavity resonances) was made [14-16]. At fixed cavity length, a Ti-sapphire laser was tuned to find successive resonant wavelengths $\left(\lambda_{1}, \lambda_{2}\right)$ of the cavity, and an experimentally determined length was then defined by $L_{\text {expt }}=\lambda_{1} \lambda_{2} / 2\left(\lambda_{1}-\lambda_{2}\right)$.

This length comprises the actual physical length between the two mirror surfaces, $L$, plus a contribution from leakage of the mode into the mirror stack, which gives rise to an additional phase shift at the coatings, to give a length $L_{\text {eff }}$ $>L$. In addition, the leakage into the coatings increases with wavelength as $\left(\lambda_{1}, \lambda_{2}\right)$ move away from the coating design wavelength, so this gives another additional contribution to the round-trip phase and hence to the measured length $L_{\text {expt }}$.

As discussed in Ref. [14], if $\lambda_{1}$ and $\lambda_{2}$ were closely spaced compared to the scale on which the coating properties vary (so that coating dispersion could be neglected), then near the design wavelength of the coating we would have $L_{\text {expt }}=L_{\text {eff }}=L+\left[1 /\left(n_{H}-n_{L}\right)\right] \times \lambda_{c} / 2$, where $n_{H}$ and $n_{L}$ are the high and low index materials of the stack, and $\lambda_{c}$

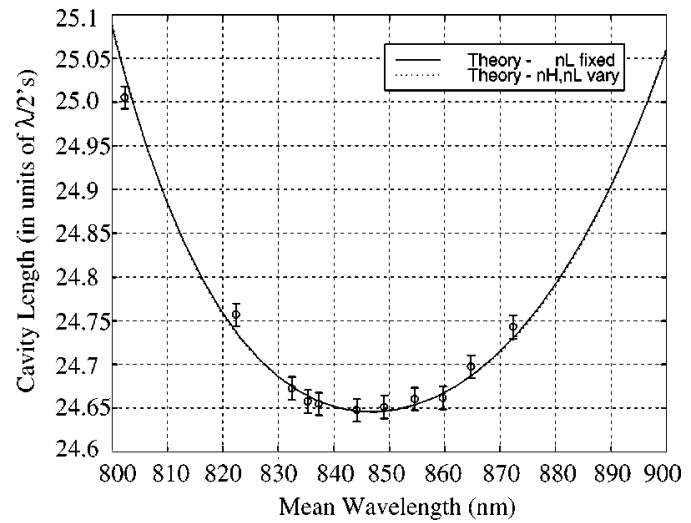

FIG. 2. The cavity length $L_{\text {expt }}$ measured from the free spectral range (FSR) varies about the design wavelength of the coating. Fitting a model to these data points gives a measure of mirror transmission (from fitting of the difference $n_{H}-n_{L}$ ) and center wavelength (from fitting layer thickness).

$=2 \lambda_{1} \lambda_{2} /\left(\lambda_{1}+\lambda_{2}\right)$ is the average (in frequency) of wavelengths $\lambda_{1}$ and $\lambda_{2}$. We thereby have a dependence of the free spectral range on $\left[1 /\left(n_{H}-n_{L}\right)\right]$, which, combined with the transmission (which depends on $n_{L} / n_{H}$ ), can fix $n_{H}$ and $n_{L}$. For these materials, this gives $L_{\text {eff }}=L+1.633 \lambda_{c} / 2$. However, for our measurements with short cavities, $\lambda_{1}$ and $\lambda_{2}$ are separated by $\simeq 30 \mathrm{~nm}$, so $L_{\text {expt }}>L_{\text {eff }}$. But we can still use the complete model to fit to the measured values $\left(\lambda_{1}, \lambda_{2}\right)$ and determine parameters of the coating. Finally, by mapping out this wavelength dependence of the free spectral range to find $\min \left(L_{\text {expt }}\right)$, we find the center wavelength of the coating.

In the model, the refractive indices used are adjusted to obtain the same pairs $\left(\lambda_{1}, \lambda_{2}\right)$ as measured. Then, the layer thickness in the model is adjusted to agree with the measured coating center wavelength. By using the additional information of the measured mirror transmission $T$ from Sec. II, we can now either (i) derive independent values for the refractive indices and layer thickness, or (ii) assuming one index is known, use the refractive indices and layer thickness information to give an independent value for the mirror transmission, which can be compared to the measurement of Sec. II.

That the dispersion (FSR) measurement alone is sufficient to determine the lossless part of the mirror properties represents some useful information for the mirror-coating technician: the index difference $n_{H}-n_{L}$ and the optical thickness of the coating layers can be simply measured in this way without interference from absorption/scatter losses. And, if $n_{L}$ is known, this also gives a simple way of finding the mirror transmission. Adding in a direct measurement of mirror transmission yields values for $n_{H}$ and $n_{L}$ separately.

Data obtained from these measurements are shown in Fig. 2 , where $L_{\text {expt }}$ is plotted as a function of wavelength, for a $10 \mu \mathrm{m}$ cavity with $10 \mathrm{~cm}$ radius of curvature mirrors. The circles are measured data and the curves are the calculation from the model, with parameters chosen to best fit the data. These data were taken by setting the cavity to a series of different lengths, and recording a pair of resonant wavelengths $\left(\lambda_{1}, \lambda_{2}\right)$ at each length. The $x$ axis is the mean wavelength $\lambda_{c}=2 \lambda_{1} \lambda_{2} /\left(\lambda_{1}+\lambda_{2}\right)$; the $y$ axis is the measured cav- 
ity length $L_{\text {expt }}=\lambda_{1} \lambda_{2} / 2\left(\lambda_{1}-\lambda_{2}\right)$ shown in units of $\lambda_{1} / 2$ : for each pair $\left(\lambda_{1}, \lambda_{2}\right)$, the length is such that $L_{\text {expt }} /\left(\lambda_{1} / 2\right)$ $=24 . x x$. Dividing by $\lambda_{2}$ instead would exactly give 23.xx, since by rearranging the formula for $L_{\text {expt }}$ we see that $L_{\text {expt }} /\left(\lambda_{1} / 2\right) \equiv L_{\text {expt }} /\left(\lambda_{2} / 2\right)+1$. Due to a finite drift in the cavity length, each measurement of $\lambda$ was made to only five digits resolution (e.g., $852.59 \pm 0.01 \mathrm{~nm}$ ), leading to the uncertainty in $L_{\text {expt }}$ shown. Uncertainty in $\lambda_{c}$ is $\pm 0.03 \mathrm{~nm}$ and cannot be seen on this scale.

Two theory curves are shown. The solid curve shows a model with $n_{L}$ assumed to be fixed at its nominal value of $n_{L}=1.455$. To best fit the data, $n_{H}$ was increased to $n_{H}$ $=2.0676$ (a factor of 1.3\%). In addition, the center wavelength was shifted to $847 \mathrm{~nm}$ (by reducing the thickness of each $\lambda / 4$ layer by $0.6 \%$ ). Discussions with REO confirmed that $1.3 \%$ is a known offset in $n_{H}$ for the particular coating machine that produced this run, and also that a few nm uncertainty in the center wavelength is typical. With these parameters, the inferred mirror transmission is $T_{\mathrm{inf}}=4.6$ $\pm 0.2 \mathrm{ppm}$, agreeing well with the measured value $T_{\exp }$ $=4.3 \mathrm{ppm}$ from Sec. II. The dotted curve (which overlaps the solid curve) shows the model when both $n_{L}$ and $n_{H}$ are allowed to vary. Their values are chosen to match both the FSR measurement shown and to give a mirror transmission to match exactly the experimentally determined value $T_{\exp }$ $=4.3 \mathrm{ppm}$. Parameters that satisfy these criteria are $n_{H}$ $=2.0564(0.75 \%$ increase $)$ and $n_{L}=1.4440(0.76 \%$ decrease). Our direct measurement of $T$ in Sec. II had a large uncertainty, which limits the absolute determination of $n_{H}$ and $n_{L}$ to about this $1 \%$ level. However, a more precise measurement could in principle determine the indices at the $0.1 \%$ level. One application might be to measure $T$ and the FSR as a function of position across a mirror substrate, thereby mapping out stress-induced variations in the refractive indices at the $0.1 \%$ level with a spatial resolution of $\sim 10 \mu \mathrm{m}$.

In this data set, the correction for the Gaussian phase difference between the actual resonator mode and the plane wave of the model has been neglected. After the propagation distance from the mode waist to the mirror surfaces, a Gaussian beam will have acquired less phase than a plane wave traveling the same distance. For a $10 \mu \mathrm{m}$ cavity with $10 \mathrm{~cm}$ radius of curvature mirrors, this gives a $2 \%$ correction, corresponding to a shift in $L_{\text {expt }}$ by $\simeq 0.0045$ cavity orders (that is, $\Delta L \simeq+(\lambda / 2) \times 0.0045)$. Lowering the refractive index contrast of the model to shift the calculated curve by this amount would increase the inferred mirror transmission by $\lesssim 0.1 \mathrm{ppm}$. For our second cavity ( $44 \mu \mathrm{m}, 20 \mathrm{~cm}$ radius of curvature mirrors), the correction is 0.0066 cavity orders.

The mirror phase shift (FSR measurement) is only sensitive to the transmission (index contrast) and center wavelength (layer thickness). Therefore, if absorption/scatter losses are added to the model (by introducing an imaginary component to the refractive index), the cavity resonance wavelengths do not change. More precisely, adding a scattering loss at the mirror surfaces has exactly zero effect on the FSR and mirror transmission. Adding losses within the coatings has a small effect: increasing the mirror absorption from
$0.5 \mathrm{ppm}$ to $2 \mathrm{ppm}$ (an experimentally reasonable range and we distribute the loss evenly to each coating layer) changes the mirror transmission by a factor of $\simeq 10^{-5} T$, clearly negligible, and again there is no effect on the FSR measurement. As a result, this measurement (with $n_{L}$ assumed fixed) provided a very simple and sensitive inference of the mirror transmission of $T_{\text {inf }}=4.6 \pm 0.2 \mathrm{ppm}$, which is unaffected by absorption/scatter losses.

The same measurement and fitting procedure was used on another cavity with mirrors from the same coating run. This $44 \mu \mathrm{m}$ cavity made from $20 \mathrm{~cm}$ radius of curvature mirrors gave a transmission of $T_{\mathrm{inf}}=4.5 \pm 0.2 \mathrm{ppm}$, with a center wavelength of $848 \mathrm{~nm}$. (This was the cavity used for the direct measurements of Sec. II, which gave $T=4.3 \mathrm{ppm}$.)

One other factor that has been ignored so far is the effect of fluctuations in the $\lambda / 4$ layer thickness. Discussions with REO suggested that a $1 \%$ variation in thickness was reasonable, so a Gaussian-distributed variation (of standard deviation 1\%) was added to the layer thicknesses of the model. For cavity calculations, identical mirrors were used for both sides of the cavity. The principal effect of this variation is to shift the center wavelength of the coating over several realizations of random coatings, this resulted in an rms shift of the center wavelength by $\pm 1.2 \mathrm{~nm}$. So, the measured shift of center wavelength in the coating (from $852 \mathrm{~nm}$ to $847 \mathrm{~nm}$ ) is probably due partly to a systematic offset and partly to fluctuations. The mirror transmission is also affected: the value of the transmission is on average increased slightly, by $0.6 \%$ in the case studied, from $4.55 \mathrm{ppm}$ to $4.58 \mathrm{ppm}$ at the center of the coating. At the level of our current measurements, this is another negligible effect, but with a more precise measurement aimed at determining $n_{H}$ and $n_{L}$, the possibility of a systematic offset from this mechanism should be considered. Lastly, the FSR measurement is mostly effected via the change in center wavelength of the coating: the value of $\min \left(L_{\text {expt(simulated })}\right)$ has a mean the same as without the added fluctuations, and varies by only 0.0014 mode orders rms, again negligible for our purposes.

Another useful result of these calculations is that the free spectral range of the cavity is well known, so that resonant wavelengths of the cavity can be accurately predicted. This is important for choosing a diode laser of correct center wavelength to match the mode, for applications such as cavity locking or dipole-force traps. With the idea of using a laser of $>920 \mathrm{~nm}$ wavelength to form an intracavity dipole-force trap [17], this knowledge was particularly important: our Ti:sapphire laser tuned only as high as $890 \mathrm{~nm}$ so cavity resonances in this wavelength range could not be measured, only predicted. With the parameters chosen above for the model, the following theoretical and experimental resonance wavelengths resulted:

$787.208,818.659,853.255,890.798,930.683 \mathrm{~nm}$ for theory;

$787.170,818.651,853.255,890.800 \mathrm{~nm}$, N/A for experiment. 

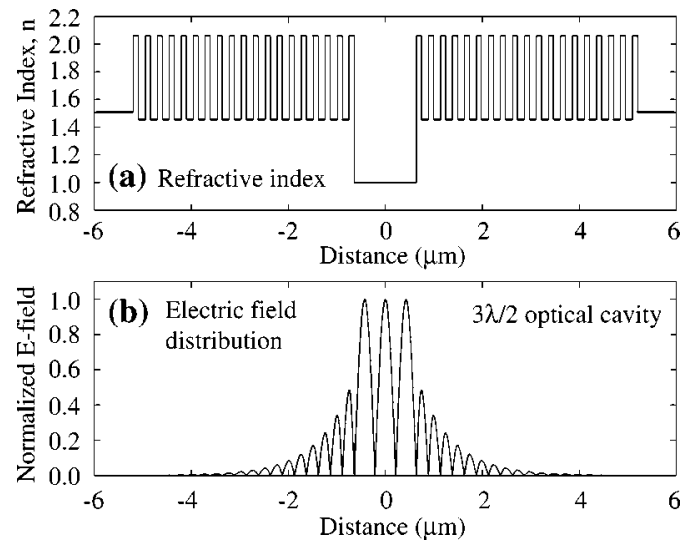

FIG. 3. (a) Mirror refractive index stack design, and (b) resulting electric-field distribution for a resonant $3 \lambda / 2$ cavity made from dielectric mirrors.

The experimental value for the cavity resonance can then confidently be predicted to be $930.7 \pm 0.05 \mathrm{~nm}$, and a diode laser chosen accordingly.

\section{LIMITATIONS TO MODE VOLUME}

In a similar calculation to the one described above, it is possible to calculate the field distribution of light inside the resonant cavity, by describing each layer separately with a left- and right-traveling plane wave, then matching electromagnetic boundary conditions between layers. An example of this kind of calculation is shown in Fig. 3, where refractive index and field distribution (modulus of the electric field) are plotted as a function of distance for a cavity with length $L_{\text {eff }}=3 \lambda / 2$. The coupling strength $g_{0}$ of an atom placed in the center of the cavity mode is proportional to $1 / \sqrt{V_{m}}$, where $V_{m}$ is the cavity mode volume found by integrating the field $(D \cdot E)$ over the standing wave and Gaussian transverse mode profile. Large coupling is achieved by making a short cavity with a small mode waist (short radius of curvature mirrors).

For a cavity of physical length $L$, the "leakage" of the mode into the $\lambda / 4$ mirror stack (look at the tails of the mode in Fig. 3) that increases $L$ to $L_{\text {eff }}$ also increases the cavity mode volume. For our materials at $852 \mathrm{~nm}, L_{\mathrm{eff}}=L$ $+1.633 \lambda / 2$, so for a cavity with physical distance between mirror surfaces $L=\lambda / 2$, the cavity mode volume ends up being 2.63 times larger than might otherwise have been expected, and hence the atom-cavity coupling $g_{0}$ is 0.6 times smaller than the naive estimate based on the physical separation of the mirror surfaces.

This effect is proportionately larger as the cavity length gets shorter. In Fig. 4, the expected $g_{0}$ is plotted for a cavity formed with two $20 \mathrm{~cm}$ radius of curvature mirrors, as a function of the physical distance $L$ between the mirrors. The two curves show a real mirror (with $g_{0}$ reduced by leakage into the coatings) and an idealized mirror with no leakage (perfect reflectors at $\pm L / 2$ ). The transverse (Gaussian waist) dimension is calculated by simple Gaussian beam propagation, which is not strictly accurate for length scales less than a few microns; however, any error in this should be roughly

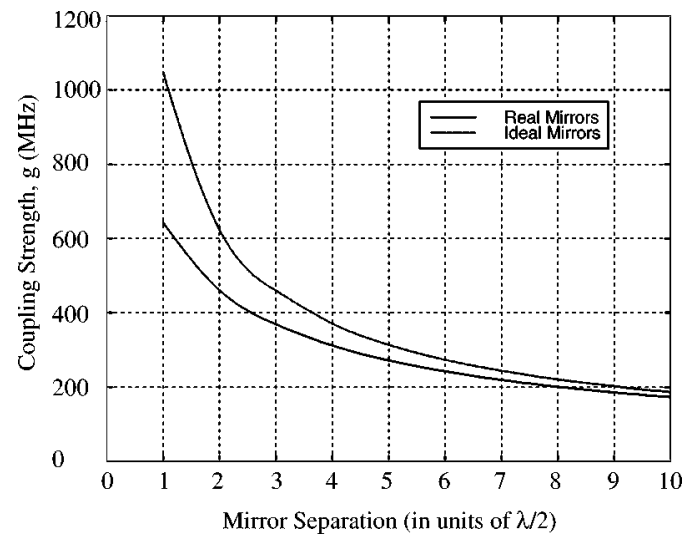

FIG. 4. Coupling coefficient $g$ (expressed in cycles per second, with $2 g$ as the single-photon Rabi frequency) versus the physical separation $L$ of the surfaces of two mirrors forming a Fabry-Perot resonator. Due to penetration of the standing-wave mode into the mirror coatings, the cavity mode volume achieved with real mirrors is larger (and hence the coupling strength smaller) than for an ideal mirror with the same spacing between mirror surfaces but no penetration.

the same for both the ideal and actual mirror cases, so the ratio of these should remain sensibly correct. The cavity is assumed resonant at an integer number of half-wavelengths of light at the $852 \mathrm{~nm}$ Cs D2 transition; that is, each $\lambda / 2$ is a distance of $0.426 \mu \mathrm{m}$.

The discrepancy between the expected and achieved coupling $g$ is large even for our longer cavities-5\% for a $10 \mu \mathrm{m}$ cavity. However, in the lab this is largely compensated for by the fact that we never measure the actual physical distance $L$ between mirror surfaces, but instead $L_{\text {expt }}$ $=\lambda_{1} \lambda_{2} / 2\left(\lambda_{1}-\lambda_{2}\right)$, which is close to $L_{\text {eff }}$, and so incorporates the same offset of mirror penetration that determines $g_{0}$. This method of length measurement breaks down eventually due to the dispersion of the mirror coatings: Eventually, if $\lambda_{1}$ is at the center of the coating, $\lambda_{2}$ will be so far separated in wavelength that it reaches the edges of the mirror coating stopband, and the observed round-trip phase has then more to do with the structure of the dielectric coatings than it does with the vacuum gap between the surfaces of the cavity mirrors. That is to say, our measured $L_{\text {expt }}$ becomes increasingly different from $L_{\text {eff }}$ and introduces an offset in estimating the mode volume as the cavity length approaches the scale of the wavelength.

At $L=20 \lambda / 2$ physical length (the regime of our present cavities), the difference between the coupling coefficient $g_{0}$ inferred from $L_{\text {expt }}$ and that found by integrating $D E$ over the mode volume is $<0.1 \%$. At $L=10 \lambda / 2(4.26 \mu \mathrm{m})$, it would be a $1 \%$ error; at $5 \lambda / 2$, an $8 \%$ error. Note, however, that knowledge of these offsets means that when calculating $g_{0}$ from $L_{\text {expt }}$, we can compensate for this effect. Measurements of $L_{\text {expt }}$ for cavities any shorter than $5 \lambda / 2$ would be impossible since $\lambda_{2}$ has reached the edge of the mirror stopband. To align shorter cavities, a new method for length measurement will need to be developed, such as measuring the frequency spacing of transverse modes.

We are now in a position to estimate parameters for the 
best Fabry-Perot cavity that will be experimentally feasible in the near future using this type of mirror. First consider a $L=\lambda / 2$ cavity with $20 \mathrm{~cm}$ radius of curvature mirrors. If the mirror transmission and losses were each reduced to $T=l$ $=0.5 \mathrm{ppm}$ to yield a cavity finesse of $\mathcal{F}=3.14 \times 10^{6}$, then this cavity has parameters $\left(g_{0}, \kappa, \gamma_{\perp}\right) / 2 \pi$ $=(647,56,2.6) \mathrm{MHz}$, which gives the critical photon number $n_{0}=\gamma_{\perp}^{2} / 2 g_{0}^{2}=8.1 \times 10^{-6}$ and the critical atom number $N_{0}=2 \kappa \gamma_{\perp} / g_{0}^{2}=7.0 \times 10^{-4}$. To make a cavity of this length, the $20 \mathrm{~cm}$ mirrors would have to be reduced to a diameter of $0.5 \mathrm{~mm}$ rather than $1 \mathrm{~mm}$. At this size, there would still be a $0.11 \mu \mathrm{m}$ gap between the mirror edges for the $L$ $=\lambda / 2(0.426 \mu \mathrm{m})$, cavity length, which should make it possible to still get atoms into and out of the cavity (as in Refs. [3-8]), and to align the mirrors.

If the mirror diameter could be reduced to $350 \mu \mathrm{m}$ (without adversely affecting the cavity losses), then a $10 \mathrm{~cm}$ radius of curvature mirrors could be used, with a $0.12 \mu \mathrm{m}$ gap at the edges. Due to the tighter radius of curvature, $g_{0} / 2 \pi$ would be increased to $770 \mathrm{MHz}$ in this case. Now speculating that "dream" mirrors of $T=0.2 \mathrm{ppm}$ transmission, $l$ $=0.2 \mathrm{ppm}$ loss might be possible $\left(\mathcal{F}=7.85 \times 10^{6}\right)$, we could aim for the ultimate goal of $\left(g_{0}, \kappa, \gamma_{\perp}\right) / 2 \pi$ $=(770,22,2.6) \mathrm{MHz}$, in which case $n_{0}=5.7 \times 10^{-6}$ photons and $N_{0}=1.9 \times 10^{-4}$ atoms.
In conclusion, we have presented two measurement approaches, one based upon direct loss and the other on cavity dispersion, that produce the same quantitative determination of the mirror-coating properties. The dispersion measurement is more informative, as it has the potential to determine the complete characteristics of a mirror. A model has been derived to link the mirror properties to the physical parameters of coating layers. Issues relevant to optical cavity QED, such as the cavity field mode structure, have been discussed.

\section{ACKNOWLEDGMENTS}

First and foremost, we thank Research Electro-Optics, Inc. for providing the best quality mirrors and coatings, which have made our work possible. In particular, the critical and ongoing contributions of R. Lalezari and J. Sandburg to our reserach program in cavity QED are gratefully acknowledged. Our Caltech colleagues David Vernooy and Theresa Lynn made important contributions to the work presented here. This work was funded by the National Science Foundation, by the Caltech MURI on Quantum Networks administered by the U.S. Army Research Office, by DARPA through the QUIC (Quantum Information and Computing) program, by the Office of Naval Research, and by the National Institutes of Standards and Technology.
[1] H.J. Kimble, in Cavity Quantum Electrodynamics, edited by P.R. Berman (Academic Press, Boston, 1994), pp. 203-266.

[2] S.J. van Enk, J.I. Cirac, and P. Zoller, Science 279, 205 (1998).

[3] H. Mabuchi, Q. Turchette, M.S. Chapman, and H.J. Kimble, Opt. Lett. 21, 1393 (1996).

[4] C.J. Hood, M.S. Chapman, T.W. Lynn, and H.J. Kimble, Phys. Rev. Lett. 80, 4157 (1998).

[5] H. Mabuchi, J. Ye, and H.J. Kimble, Appl. Phys. B: Lasers Opt. 68, 1095 (1999).

[6] J. Ye, C.J. Hood, T.W. Lynn, H. Mabuchi, D.W. Vernooy, and H.J. Kimble, IEEE Trans. Instrum. Meas. 48, 608 (1999).

[7] J. Ye, D.W. Vernooy, and H.J. Kimble, Phys. Rev. Lett. 83, 4987 (1999).

[8] C.J. Hood, T.W. Lynn, A.C. Doherty, A.S. Parkins, and H.J. Kimble, Science 287, 1447 (2000).

[9] P.W.H. Pinkse, T. Fischer, P. Maunz, and G. Rempe, Nature (London) 404, 365 (2000).

[10] J.J. Childs, K. An, M.S. Otteson, R.R. Dasari, and M.S. Feld,
Phys. Rev. Lett. 77, 2901 (1996); K. An and M.S. Feld, Phys. Rev. A 56, 1662 (1997).

[11] G. Rempe, R. J. Thompson, H. J. Kimble, and R. Lalezari, Opt. Lett. 17, 363 (1992).

[12] Research Electro-Optics, Inc., 1855 South 57th Court, Boulder, Colorado 80301.

[13] E. Hecht, Optics (Addison-Wesley Publishing, Reading, MA, 1987), pp. 373-375.

[14] For a derivation of the $\left[1 /\left(n_{H}-n_{L}\right)\right]$ effective length correction, see R.G. DeVoe, C. Fabre, K. Jungmann, J. Hoffnagle, and R.G. Brewer, Phys. Rev. A 37, 1802 (1988).

[15] W. Lichten, J. Opt. Soc. Am. A 2, 1869 (1985).

[16] H. P. Layer, R. D. Deslattes, and W.G. Schewietzer, Jr., Appl. Opt. 15, 734 (1976).

[17] H. J. Kimble, C. J. Hood, T. W. Lynn, H. Mabuchi, D. W. Vernooy, and J. Ye, in Laser Spectroscopy XIV, edited by R. Blatt, J. Eschner, D. Leibfried, and F. Schmidt-Kaler (World Scientific, Singapore, 1999), p. 80. 